\title{
Norois
}

Environnement, aménagement, société

\section{Croissance démographique, pression foncière et insertion territoriale par les abattis en Guyane française}

Population growth, land pressure and territorial insertion using shifting cultivation in French Guiana

Moïse Tsayem Demaze

\section{OpenEdition}

Journals

Édition électronique

URL : http://journals.openedition.org/norois/1131

DOI : $10.4000 /$ norois. 1131

ISBN : 978-2-7535-1554-3

ISSN : $1760-8546$

Éditeur

Presses universitaires de Rennes

\section{Édition imprimée}

Date de publication : 1 mars 2008

Pagination : 111-127

ISBN : 978-2-7535-0690-9

ISSN : 0029-182X

Référence électronique

Moïse Tsayem Demaze, «Croissance démographique, pression foncière et insertion territoriale par les abattis en Guyane française », Norois [En ligne], 206 | 2008/1, mis en ligne le 01 mars 2010, consulté le 30 avril 2019. URL : http://journals.openedition.org/norois/1131; DOI : 10.4000/norois.1131

(c) Tous droits réservés 


\title{
Croissance démographique, Pression foncière
}

\section{ET INSERTION TERRITORIALE PAR LES ABATTIS EN GUYANE FRANÇAISE}

\author{
MoÏse Tsayem Demaze \\ UMR ESO 6590 CNRS, GREGUM - chercheur associé à l'IRD, US 140 ESPACE \\ (Université du Maine), \\ Avenue Olivier-Messiaen - 72085 LE MANS cedex \\ Moise.Tsayem_Demaze@univ-lemans.fr
}

\section{RÉSUMÉ}

La Guyane française connaît une forte croissance démographique depuis plusieurs décennies. Sa population est passée de 23000 habitants en 1946 à environ 200000 habitants en 2007, soit une multiplication par 8 en 60 ans. Cette forte croissance démographique est sous-tendue par un solde naturel positif mais aussi par l'attraction migratoire que la Guyane, territoire français et européen, exerce sur ses voisins sud-américains et caribéens (Suriname, Brésil, Haïti, etc.). L'augmentation continue de la population entraîne une pression foncière et un accroissement des besoins en terre d'autant plus importants que la grande majorité du territoire, près de $90 \%$, est occupée par la forêt dense qui appartient au domaine privé de l'État. Dans ce contexte, faire un abattis, c'est-à-dire défricher une petite portion de forêt et la mettre en culture, parfois de manière clandestine, devient un moyen d'insertion territoriale surtout pour les nouveaux arrivants. Cette situation s'observe par exemple dans le Nord-Ouest de la Guyane où des populations en provenance notamment du Suriname s'appuient sur les abattis pour avoir un ancrage territorial afin de s'insérer ensuite dans les circuits socio-économiques. À partir d'observations de terrain, illustrées par des photos prises au sol, complétées par des supports issus du traitement des photographies aériennes et d'images satellites, cet article présente cette forme de territorialisation et ses implications sur la gestion du foncier et sur l'aménagement du territoire. Les statistiques issues des recensements de la population et de l'agriculture sont utilisées pour décrire l'évolution de la population et la pression foncière consécutive à l'accroissement des besoins en terres agricoles.

MotS CLÉS : Abattis - Démographie - Foncier - Guyane française - Territoire.

\section{ABSTRACT \\ Population growth, land pressure and territorial insertion using shifting cultivation in French Guiana}

Since several decades, the population of French Guiana is increasing strongly. This population has been multiplied by 8 in 60 years: 23000 inhabitants in 1946 and approximately 200000 in 2007. This high demographic growth is due to a positive natural balance and to the attraction that French Guiana, French and European territory, exerts on his South American and Caribbean neighbours (Surinam, Brazil, Haiti, etc). The continuous increase of population involves land pressure since the great majority of the territory, nearly $90 \%$, is occupied by the dense forest which belongs to the private property of the State. In 
this context, the fact of clearing a small portion of forest and cultivate it, sometimes in a clandestine way, can become a mean of territorial insertion especially for the new arrivals. This situation is observed for example in the North-West of French Guiana where populations coming notably from Surinam are using shifting cultivation called locally "abattis" to have a territorial insertion in order to later integrate local socio-economic circuits. Fields observations illustrated by photographs taken on the ground and supplemented by supports resulting from air photographs and satellite images permit us to present this form of territorialisation and its implications on land management and territory planning. Statistics resulting from population and agricultural censuses are used to describe demographic growth and the increasing land pressure and needs for agricultural lands.

\footnotetext{
KEY WORDS : Demography - French Guiana - Land tenure - Shifting Cultivation - Territory.
}

De 23000 personnes au moment de la départementalisation en 1946, le nombre d'habitants en Guyane française a atteint 191000 en 2005 (INSEE-ACSE, 2006) et probablement 200000 en 2007. La densité démographique a franchi le seuil de 2 personnes par $\mathrm{km}^{2}$ puisque la superficie de la Guyane est de $84000 \mathrm{~km}^{2}$. Mais la population ne dispose en principe que de $10 \%$ de cette étendue car $90 \%$ du territoire appartient au domaine de la forêt dense amazonienne (fig. 1) qui est la propriété privée de l'État. L'augmentation continue de la population est consécutive au solde naturel positif (forte natalité associée à une mortalité relativement faible) mais aussi aux flux migratoires qui apportent à la Guyane française près du tiers de sa population totale.

La croissance démographique suscite une pression foncière caractérisée par une pratique plus ou moins formelle d'insertion socio-spatiale par des défrichements agricoles traditionnels souvent en marge de la réglementation foncière. Il s'agit de la pratique agricole appelée localement abattis. Technique agricole ancestrale des Amérindiens adoptée par les autres communautés installées en Guyane plus récemment, l'abattis semble jouer de plus en plus un rôle d'insertion territoriale des populations au-delà de sa fonction d'autosubsistance alimentaire. En attendant une amélioration de leur situation, de nombreux immigrés ouvrent des abattis pour marquer leur présence et pour satisfaire leurs besoins alimentaires par la production agricole dont une partie peut éventuellement être vendue. Cette situation prévaut notamment dans le Nord-Ouest de la Guyane où débarquent de nombreux immigrés en provenance du Suriname ou de Haïti (Gachet et al., 1993).

Les observations de terrain faites en 2000, 2005 et 2006, nous permettent de décrire ce processus de territorialisation par les abattis en le situant dans le contexte général de croissance démographique qui engendre et accentue localement la pression foncière en Guyane française. Les photos prises au sol, ainsi que les données de télédétection (extrait de photographies aériennes et d'images satellites), illustrent notre propos. Les données statistiques ${ }^{1}$ relatives à la population et à l'agriculture sont issues des recensements officiels effectués par l'INSEE pour la population et par la direction départementale de l'Agriculture et des Forêts (DDAF) de Guyane pour l'agriculture.

\section{De l'enfer vert à un territoire convoité ?}

La Guyane française est souvent qualifiée de désert humain ou « enfer vert » en raison de l'immensité et de la densité de sa végétation forestière considérée comme facteur explicatif du faible peuplement. Il convient de nuancer cette image dans la mesure où depuis une cinquantaine d'années, la population de ce département français d'outre-mer croit considérablement.

1. On estime que ces données sont sous-estimées car elles ne prennent pas en compte le caractère clandestin d'une partie de l'immigration et d'une partie de l'agriculture de type abattis (Manusset, 1999). 


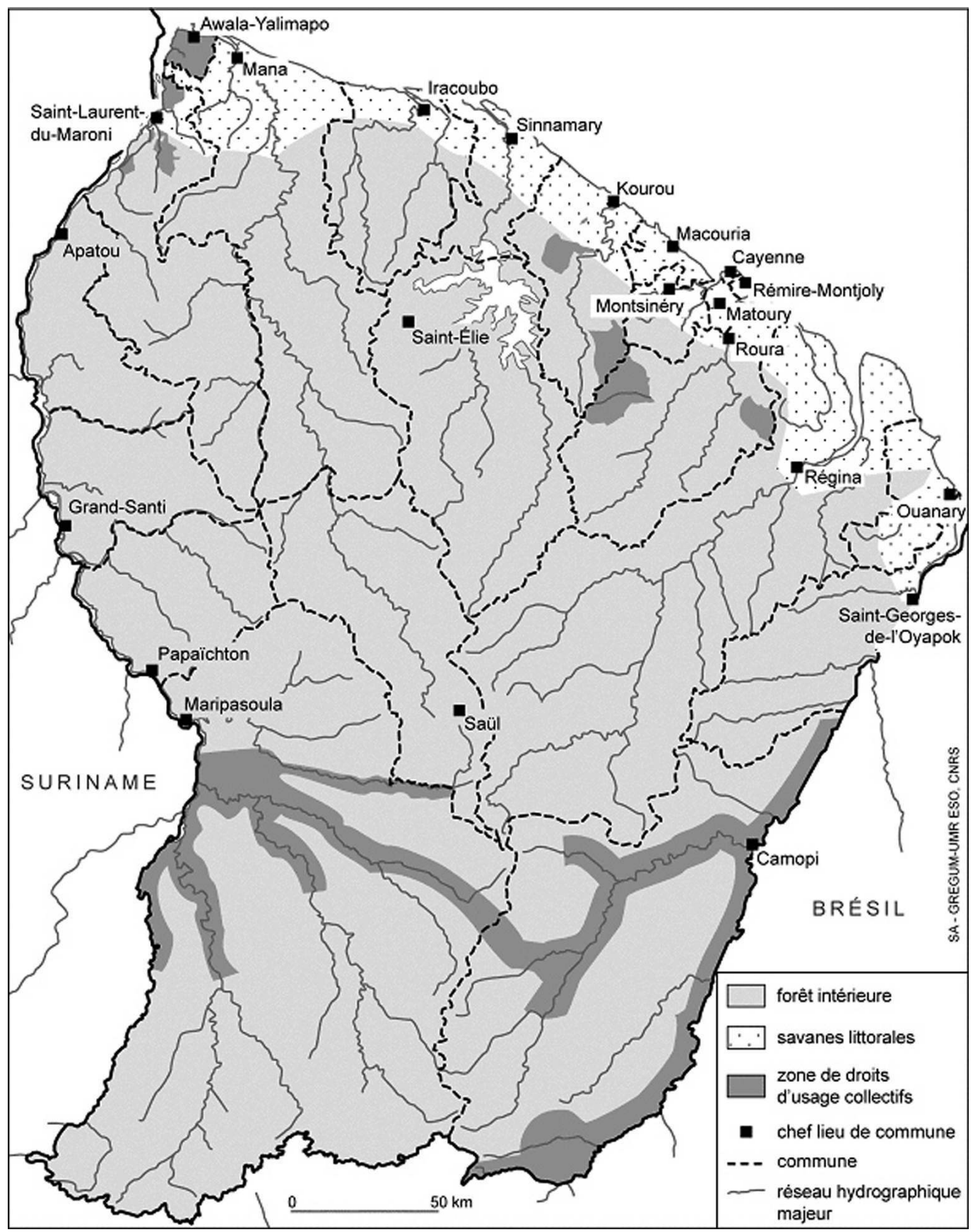

Figure 1 : L'organisation du territoire guyanais : communes, forêt, savanes, zones de droit d'usage collectif The organization of French Guiana territory: forest, savannas, areas of collective use 


\section{L'AUGMENTATION CONTINUE DE LA POPULATION}

D'après les recensements officiels effectués par l'INSEE, le nombre d'habitants est passé de 23000 en 1946 à 157000 en 1999 (tableau 1). Estimée à 191000 personnes en 2005 (INSEEACSE, 2006), la population de la Guyane a sans doute franchi le seuil de 200000 personnes en 2007. Cette forte croissance démographique a été particulièrement remarquable entre 1982 et 1990, le nombre d'habitants étant passé de 73000 à 114000 , soit une croissance annuelle de $5,7 \%$ (fig. 2). Depuis 1990, ce taux annuel connaît une inflexion qui l'a ramené entre 3,7 \% et 3,5\% (tableau 1). La croissance démographique de l'ensemble de la France (métropole et outre mer) a été de 0,5\% par an entre 1999 et 2004. Avec un taux de 3,5\% par an entre 1999 et 2005, la Guyane est la région française qui connaît la croissance démographique la plus rapide.

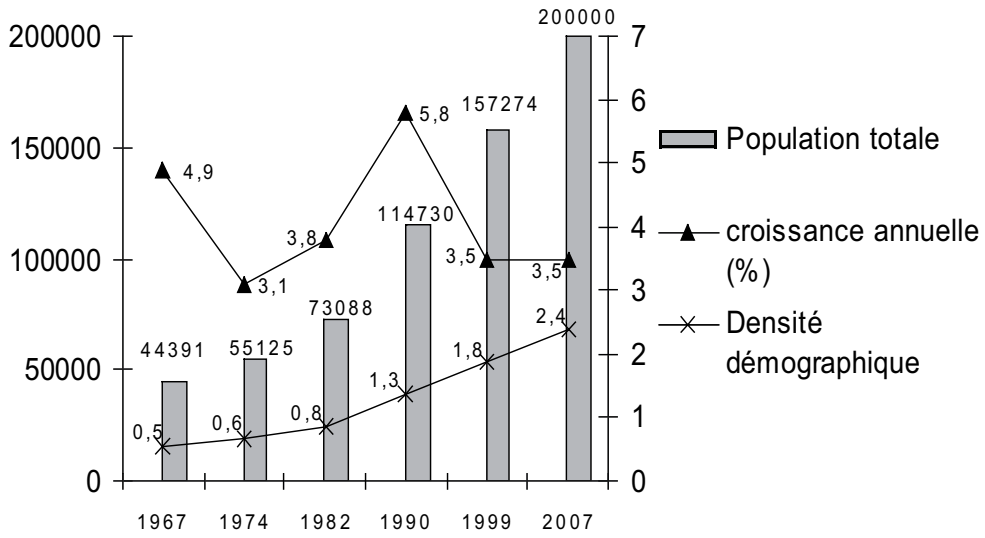

Figure 2 : Croissance démographique et augmentation des densités de population en Guyane française Demographic growth and population densities increasing in French Guiana

\begin{tabular}{|l|l|l|l|l|l|l|l|}
\hline Années & 1946 & 1967 & 1974 & 1982 & 1990 & 1999 & 2005 \\
\hline Population totale & 23298 & 44391 & 55125 & 73088 & 114730 & 157274 & 191000 \\
\hline croissance annuelle (\%) & & 4,9 & 3,1 & 3,8 & 5,8 & 3,5 & 3,5 \\
\hline Densité démographique & 0,27 & 0,53 & 0,65 & 0,87 & 1,37 & 1,88 & 2,24 \\
\hline
\end{tabular}

Tableau 1 : Évolution de la population et des densités démographiques entre 1946 et 2005 en Guyane française (Sources : Tsayem, 2002; INSEE-ACSE, 2006)

Evolution of population and demographic densities between 1946 and 2005 in French Guiana

Ce sont surtout les communes situées sur la bande côtière (fig. 1) qui ont vu leur population augmenter très significativement jusqu'en 1990. C'est en particulier le cas des communes de Cayenne, Rémire-Montjoly et Matoury (communes de la presqu'île de Cayenne) qui comptaient près de $54 \%$ de la population totale de la Guyane au recensement de 1999, sur moins de $3 \%$ du territoire. La population de Matoury est passée de 2532 à 10150 personnes entre 1982 et 1990. Tonate-Macouria, Kourou, Mana et Saint-Laurent ont connu une évolution similaire. L'ensemble des communes de la bande littorale rassemble environ $70 \%$ de la population de la Guyane, dans des territoires peu étendus, à l'instar de la commune de Cayenne qui a une surface de 23,6 km² et abrite pourtant à peu près le tiers de la population totale du département.

Depuis 1990, les communes de la zone forestière intérieure enregistrent une forte croissance démographique, même si, en valeur absolue, elle n'est pas très importante. La population de Papaïchton est passée de 740 habitants en 1990 à 1645 en 1999, soit du simple au double, comme à Maripasoula où on a dénombré 1760 personnes en 1990 et 3699 personnes en 1999. 


\section{ENTRE SOUS PEUPLEMENT GLOBAL ET PETITES AGGLOMÉRATIONS LOCALES}

Les densités démographiques donnent de la Guyane française l'image d'une région sous peuplée en dépit de l'accroissement démographique observé ces dernières décennies. Au niveau global du département, on compte actuellement un peu plus de deux habitants au $\mathrm{km}^{2}$. La densité démographique moyenne a évolué parallèlement à l'augmentation de la population totale. On est passé d'une moyenne départementale de 0,27 habitant au km² en 1946 à 1,88 en 1999 et 2,44 en 2007 (fig. 2). À titre de comparaison, le Suriname, pays voisin de la Guyane française, a une densité moyenne de 4 habitants au $\mathrm{km}^{2}$.

Que ce soit au niveau des communes ou de l'ensemble du territoire, les densités de population ont très peu de signification réelle car les zones habitées et anthropisées ne représentent qu'environ $10 \%$ de la superficie du territoire. Cette situation est similaire à celle de l'Amazonie brésilienne et pose la question de la représentation cartographique du peuplement dans un contexte où l'emprise ferestière est prédominante (Le Tourneau, 2002). Les chiffres illustrent la faible pression humaine globale sur le milieu. Mais celle-ci est variable, car les communes de la zone littorale présentent les plus fortes densités tandis que celles de la zone forestière intérieure, pourtant plus étendues, sont habitées par des populations numériquement très faibles. Au recensement général de 1999, on a dénombré dans la commune de Maripasoula, la plus grande de Guyane (et de toute la France), près de 3700 habitants sur $18360 \mathrm{~km}^{2}$, soit une densité de 0,2 habitant au $\mathrm{km}^{2}$.

En réalité, dans les communes, la population est généralement concentrée soit dans les villes (pour la zone littorale), soit dans les bourgs à partir desquels l'occupation et l'utilisation du sol sont organisées. Les villes et les bourgs sont des lieux où les densités sont élevées et où l'utilisation du sol est intense. Au fur et à mesure qu'on s'éloigne de ces lieux de concentration humaine, les densités diminuent très fortement et deviennent nulles au-delà d'une certaine distance (3 à $4 \mathrm{~km}$ ). Concentration de la population dans de petites agglomérations locales et sous peuplement global, tel semble être le reflet de la distribution dissymétrique de la population de la Guyane française. Dès lors, les dynamiques démographiques et leurs transcriptions spatiales perceptibles au niveau local, ont du mal à transparaître au niveau du département considéré globalement.

\section{UNE CROISSANCE DÉMOGRAPHIQUE SOUS-TENDUE} PAR UNE NATALITÉ ET UNE IMMIGRATION ÉLEVÉES

La natalité et l'immigration élevées sont les principaux facteurs qui expliquent la forte croissance démographique de la Guyane française. Depuis 2000, on enregistre chaque année un peu plus de 5000 naissances en Guyane contre un peu moins de 1000 décès, soit un solde naturel supérieur à 4000 personnes (tableau 2). L'indicateur conjoncturel de fécondité fait état de près de 4 enfants par femme (IEDOM, 2004). Entre 1990 et 2003, le taux d'accroissement naturel de la population est resté supérieur à $25 \%$. Cet accroissement naturel est responsable de près de $75 \%$ de la croissance démographique de la Guyane française, le reste étant dû au solde migratoire (IEDOM, 2004).

\begin{tabular}{|l|l|l|l|l|l|}
\hline & 1990 & 2000 & 2001 & 2002 & 2003 \\
\hline naissances & 3611 & 5149 & 5137 & 5276 & 5400 \\
\hline décès & 595 & 634 & 678 & 665 & 750 \\
\hline solde naturel & 3016 & 4515 & 4459 & 4616 & 4650 \\
\hline taux brut de natalité (\%o) & 31,5 & 31,4 & 30,3 & 30,1 & 29,8 \\
\hline taux brut de mortalité (\%o) & 5,2 & 3,9 & 4 & 3,8 & 4,1 \\
\hline taux d'accroissement naturel (\%o) & 26,3 & 27,5 & 26,3 & 26,3 & 25,7 \\
\hline indice de fécondité & & 4 & 3,9 & 3,9 & 3,9 \\
\hline
\end{tabular}

Tableau 2 : Évolution récente de quelques indicateurs démographiques de la Guyane française (Source : IEDOM, 2004) Recent evolution of some demographic indicators of French Guiana 
Avec 46100 personnes au recensement de 1999, les immigrés constituaient 26,6 \% de la population de la Guyane française (INSEE-ACSE, 2006), faisant ainsi de la Guyane la région française dans laquelle le taux d'immigrés est le plus élevé (devant l'Ile-de-France et la Corse avec respectivement $15 \%$ et $10 \%$, la moyenne nationale étant de $7 \%$ ). Le taux de croissance de la population immigrée est passé de $2 \%$ par an entre 1990 et 1999 à $4 \%$ par an entre 1999 et 2005 (INSEE-ACSE, 2006). La part des immigrés dans la population totale a dépassé le seuil de $30 \%$ au recensement de 1990 (tableau 3). Ce maximum s'explique par l'arrivée en Guyane française à la fin des années 1980 de populations fuyant la guerre civile qui s'est déroulée au Suriname entre 1982 et 1992. Estimées à près de 15000 personnes (Piantoni, 1998 et 1999), ces populations avaient reçu le statut de PPDS (Personnes Provisoirement Déplacées du Suriname). Elles ont été accueillies essentiellement dans le nord-ouest de la Guyane, dans la localité de Charvein près de Saint-Laurent-du-Maroni. Après une décrue relative entre 1990 et 1999, la part des immigrés dans la population guyanaise a approché le taux de $30 \%$ en 2005 (tableau 3). Le Suriname, Haïti et le Brésil sont les trois premiers pourvoyeurs de l’immigration en Guyane française (tableau 3). Par son appartenance à la France et à l'Union Européenne, la Guyane française exerce une attraction migratoire sur les pays voisins. Un immigré sur trois vient du Suriname (INSEE-ACSE, 2006). Ces données statistiques ne tiennent pas compte de l'immigration clandestine assez importante en Guyane en raison de la fluidité et de l'étendue considérable des frontières.

\begin{tabular}{|l|l|l|l|l|l|}
\hline & 1974 & 1982 & 1990 & 1999 & 2005 \\
\hline population totale & 57348 & 73012 & 114808 & 156790 & 191000 \\
\hline immigrés & 6412 & 18803 & 34923 & 41649 & 55390 \\
\hline part des immigrés (\%) & 11,2 & 25,8 & 30,4 & 26,6 & 29 \\
\hline originaires du Suriname (\%) & 19,4 & 15,9 & 38,2 & 33,1 & \\
\hline originaires d'Haïti (\%) & 7,4 & 29 & 24 & 28,2 & \\
\hline originaires du Brésil (\%) & 24,2 & 17,9 & 15,5 & 16,8 & \\
\hline
\end{tabular}

Tableau 3 : Importance de l'immigration dans la population de la Guyane française (Source : INSEE-ACSE, 2006) Part of immigrants in the population of French Guiana

Qu'elle soit légale ou clandestine, l’immigration, associée à la forte natalité, engendre une croissance démographique qui entraine une pression foncière. Dans ce contexte, les défrichements agricoles, et notamment les abattis, se multiplient et apparaissent comme un mode d'insertion territoriale et de pression foncière révélatrice des difficultés de gestion du foncier.

\section{L'abattis comme moyen d'insertion territoriale et comme symptôme des problèmes fonciers}

L'abattis est une variante de l'agriculture itinérante sur brûlis pratiquée par la quasi-totalité des populations qui vivent en milieu forestier guyanais. C'est une pratique agricole qui remonte à l'époque précolombienne, c'est-à-dire avant le XVI ${ }^{\mathrm{e}}$ siècle. Les communautés amérindiennes, autochtones de l'Amazonie, auraient commencé à pratiquer cette forme traditionnelle d'agriculture en Guyane française il y a maintenant 3000 ans (Grenand et Grenand, 1997). Elle a été ensuite adoptée par les communautés installées en Guyane après les Amérindiens (Noirs Marrons, Créoles, Haïtiens, Surinamais, Brésiliens). Elle consiste à mettre en culture une petite portion de forêt préalablement défrichée et brûlée (Tsayem, 2002). Le terme abattis fait référence à l'abattage des arbres sur la portion de forêt à défricher et à mettre en culture. Dans le contexte de forte immigration actuelle en Guyane française, faire un abattis s'apparente au souhait pour de nombreux immigrés de marquer leur présence dans un lieu et de pouvoir s'insérer dans ce lieu en cultivant l'abattis pour satisfaire les besoins de subsistance. L'abattis est alors la première étape dans le 
processus d'installation et d'ancrage territorial. Dès lors qu'on a marqué sa présence par le biais de l'abattis, on peut ensuite construire son carbet (case) et s'insérer dans les circuits socio-économiques locaux. L'abattis apparaît ainsi à la base ou au centre d'un processus de territorialisation au sens où « le territoire est alors vécu comme le lieu où l'on est, pas forcément celui où l'on est né, mais dans tous les cas celui où l'on vit et où l'on crée les liens qui placent le système familial en équilibre au sein d'un système local » (Moine, 2006). Très répandu actuellement au sein de la communauté des géographes (Moine, 2006; Fournier, 2007; Hérin, 2007; Keerle et Séchet, 2007), le concept territoire, ou celui de territorialisation, semble approprié pour une description de la mise en œuvre des abattis par les immigrés en Guyane. En dépit des définitions parfois divergentes (Fournier, 2007; Moine, 2006), un consensus se dégage pour considérer que le territoire, ou la territorialisation, se caractérise par l'appropriation, la gestion et l'aménagement d'un espace. Ce processus laisse des traces ou des marques qui traduisent la manière par laquelle les espaces sont appropriés, gérés et aménagés. D’après Ripoll et Veschambre (2005), les marques de l'appropriation de l'espace sont caractéristiques des inégalités sociales et des rapports de force ou de pouvoir entre des groupes sociaux.

\section{LA TERRITORIALISATION PAR LES ABATTIS}

En tant que pratique traditionnelle des populations autochtones, l'abattis est demeuré inféodé et inséré dans le territoire guyanais en particulier dans les zones de droit d'usage collectif (fig. 1). Il s'agit d'espaces concédés aux communautés d'habitants qui vivent des ressources qu'elles tirent traditionnellement de la forêt. Ces populations ont collectivement le droit d'utiliser ces espaces pour la pratique des activités traditionnelles comme l'abattis, la chasse et la cueillette. L'octroi des zones de droit d'usage collectif est régi par un décret de 1987 (code du Domaine de l'État) réaffirmé par un décret de 1992 ainsi que par le code forestier de la Guyane française adopté en 2005. Les zones de droit d'usage collectif totalisent actuellement 670000 ha et les endroits voués à l'agriculture sont consacrés essentiellement aux abattis des communautés d'Amérindiens et de Noirs Marrons (Poissonnet et al., 2006). Les zones de droit d'usage collectif sont une forme de territorialisation qui formalise et matérialise l'appropriation et la gestion collective des espaces. Mais cette forme de territorialisation, communautaire, ne concerne que les groupes de populations organisées et reconnues par l'État.

Pour les autres et en particulier pour les immigrés souvent en situation de clandestinité, la mise en place des abattis relève d'opérations spontanées d'occupation du sol. Il s'agit généralement de défrichements non planifiés suivis de la construction de cabanes ressemblant aux bidonvilles. Par ce procédé, de nombreux immigrés essayent de s’insérer et de subsister en Guyane. C'est le cas, par exemple, de certains ressortissants de la communauté haïtienne vivant à Cayenne et à Matoury (photos la à 1f). Boîtes à lettres en bordure de la rue (photos 1b, le, 1f), taudis clôturés à proximité des abattis (photos 1a, 1c, 1d), sont les signes ou les marques de cette forme d'appropriation de l'espace et d'insertion territoriale. Ces signes et marques qui contrastent nettement avec les formes d'urbanisation officielle planifiée (boites à lettres discrètes, maisons bien construites avec des matériaux de haute qualité, maisons desservies par des rues en bon état, jardins et autres espaces verts bien entretenus) sont symptomatiques des inégalités sociales et des rapports de pouvoir largement en défaveur des communautés d’immigrés qui vivent dans ces quartiers spontanés.

Le Nord-Ouest de la Guyane (communes d'Awala-Yalimapo, Saint-Laurent-du-Maroni, Apatou, Grand-Santi) offre de nombreux exemples de territorialisation par le truchement des abattis, sans doute en lien avec la présence importante des immigrés qui représentent un tiers de la population des communes de cette partie de la Guyane (INSEE-ACSE, 2006). Ces immigrés viennent principalement du Suriname, du Brésil, du Guyana et d'Haïti. Les photographies aériennes (photo 2) et les images satellites (photo 3) illustrent les formes d'abattis et les étapes de l'insertion territoriale par les défrichements agricoles. L'ouverture d'une piste principale dans la forêt donne le coup d'envoi des défrichements qui se propagent ensuite le long des pistes secondaires (photo 2b). Il 


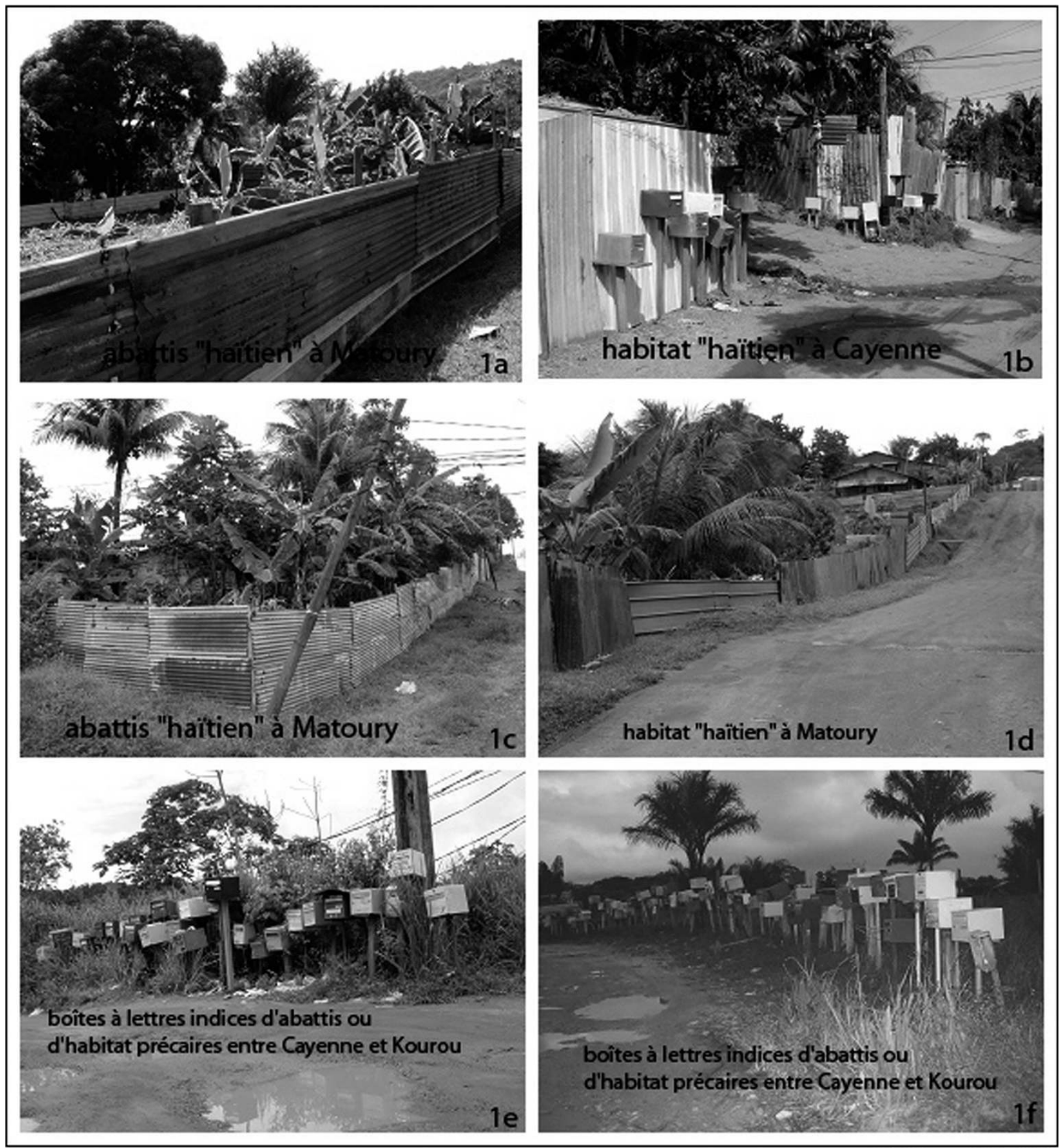

Clichés Tsayem 2005 et 2006

Photo la-lf : Insertion territoriale de type haïtien à Cayenne, Matoury et Macouria Territorial insertion of Haitian in Cayenne, Matoury and Macouria

en est de même le long des cours d'eau ou autour des bourgs, d'où la pression foncière apparente localisée autour de ces endroits (photo 2a). Cette situation illustre bien la concentration de la population autour des bourgs et le long de quelques routes, ce qui explique des densités démographiques élevées dans et autour de certaines agglomérations alors que le reste du territoire, recouvert essentiellement de forêt, présente de très faibles densités démographiques. Compte tenu de la taille individuelle des abattis et de l'étroitesse des territoires dans lesquels les défrichements sont effectués, la photographie aérienne se présente comme un outil adapté à l'observation de cette pratique d'insertion territoriale (photo $2 \mathrm{a}$ et b). Le caractère analogique (en couleurs naturelles) et la résolution de l'image (quelques décimètres) conviennent à l'interprétation visuelle et à la 

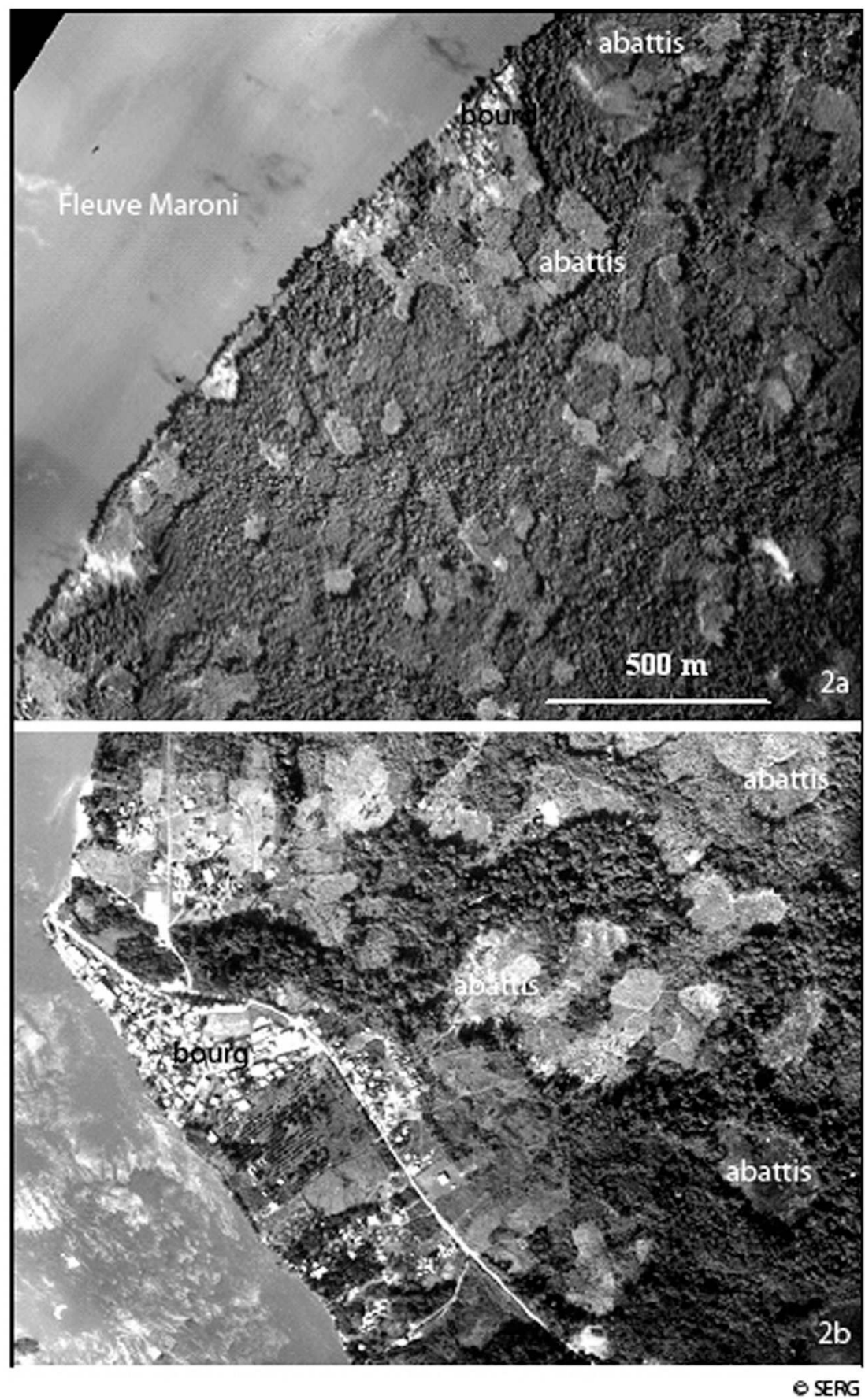

Photo 2a-2b : Paysage d'abattis sur la rive française du fleuve Maroni (frontalier avec le Suriname)

frontier) 
cartographie de détail. Ce type de document autorise la reconnaissance du stade de croissance des cultures, la distinction des différents types de couverts végétaux et la cartographie des limites de chaque abattis (Tsayem et al., 2002). De la sorte, les surfaces et périmètres sont déterminés et servent au calcul d'indices permettant l'appréciation du degré de morcellement de la forêt ainsi que le suivi de l'évolution des défrichements dans une optique d'aménagement du territoire et de gestion du foncier.

Les images satellites en compositions colorées ${ }^{2}$ (photo 3a à 3d), acquises en 1976, 1987, 1992 et 1999, offrent les mêmes perspectives d'analyse que les photos aériennes malgré la qualité médiocre des images liée aux conditions atmosphériques (nuages et leurs ombres). Les compositions colorées d'images satellites mettent en évidence la diversité des états de surface des abattis ainsi que l'évolution des défrichements dans un périmètre d'environ $2 \mathrm{~km}$ autour des routes, au cours de deux décennies. Ce périmètre de $2 \mathrm{~km}$ correspond globalement au pourtour dans lequel des immigrés du Suriname arrivés dans la localité de Charvein dans les années 1980 avaient été autorisés tacitement par l'administration guyanaise à ouvrir des abattis (photo 3b). La localisation des abattis dans ce périmètre, comme le montre les images satellites, témoigne de la pression démographique et agricole qui s'exerce uniquement dans ce pourtour (photos $3 c$ et $3 \mathrm{~d}$ ). Les états de surface des parcelles correspondent à différentes étapes d'anthropisation et de mise en culture des parcelles défrichées (sols nus, abattis denses avec des cultures au maximum de leur croissance, abattis peu denses avec des cultures en début de croissance, forêt secondaire, forêt dense, cours d'eau, etc). L'emprise des défrichements et son évolution entre 1976 et 1999, sont bien mises en évidence et peuvent être cartographiées à partir de la classification des images (Tsayem et al., 2001).

Cet exemple dans la localité de Charvein, à quelques kilomètres à l'est de Saint-Laurent-duMaroni et au sud de Mana, illustre bien le processus d'insertion territoriale par les abattis. Dans cette localité vivent près de 200 familles rassemblant environ 1000 personnes de l'ethnie Djuka (Tsayem, 2002). Elles sont installées de part et d'autre des chemins départementaux 9 et 10. Elles faisaient parti des PPDS arrivées du Suriname à partir 1986 à la suite de la guerre civile déclenchée dans ce pays. À l'issue du règlement du conflit en 1991, elles n'ont pas souhaité retourner au Suriname (Piantoni 1998 et 1999; Piantoni, 2002). L'agriculture de type abattis est devenue pour elles un moyen de subsistance et d'insertion territoriale. Bien que mis en œuvre selon le processus classique de défriche-brûlis, les abattis de cette communauté sont plus diversifiés, tant en ce qui concerne les techniques de semis que les types de cultures (photos 4a à 4d). La taille moyenne des parcelles défrichées est d'environ 0,8 ha (photos $4 \mathrm{c}$ et $4 \mathrm{~d}$ ), alors que dans les autres communautés de la Guyane, elle est généralement proche de 0,5 ha (Tsayem, 2002). Cette différence s'explique par le nombre relativement élevé d'enfants par famille Djuka, et par le souci de commercialiser une partie de la production agricole. Les parcelles de maïs côtoient celles de patates douces, de bananiers, d'ignames et de manioc (photos $4 \mathrm{c}$ et $4 \mathrm{~d}$ ). Parfois, le maïs est semé sur la même parcelle que la patate douce, le manioc et le bananier. Les jardins familiaux et les vergers sont nombreux dans la localité. Les jardins contiennent les palmiers, tandis que les vergers sont constitués essentiellement de citronniers.

La chambre d'agriculture de la Guyane a mis en place depuis quelques années un programme qui permet d'encadrer cette agriculture de subsistance en accompagnant les immigrés dans les démarches administratives nécessaires à leur installation légale en Guyane française. C'est le PEAFOG (Programme d'Encadrement de l'Agriculture Familiale dans l'Ouest Guyanais). Il bénéficie de financements de l'Europe (FEOGA : Fonds Européens d'Orientation et de Garantie Agricole), du ministère de l'Agriculture, de la Région Guyane et de la Communauté des Communes de l'Ouest Guyanais [www.guyane.chambagri.fr]. L'aide apportée par ce programme va bien au-delà de la seule dimension agricole. Elle intègre une dimension relative au développement socio-éco-

2. Ces compositions colorées associent, pour les images TM et Spot, les canaux du moyen infrarouge, du proche infrarouge et du rouge dans l'ordre colorimétrique rouge, vert, bleu. Pour les images MSS, elles associent les deux canaux de l'infrarouge et le rouge. 


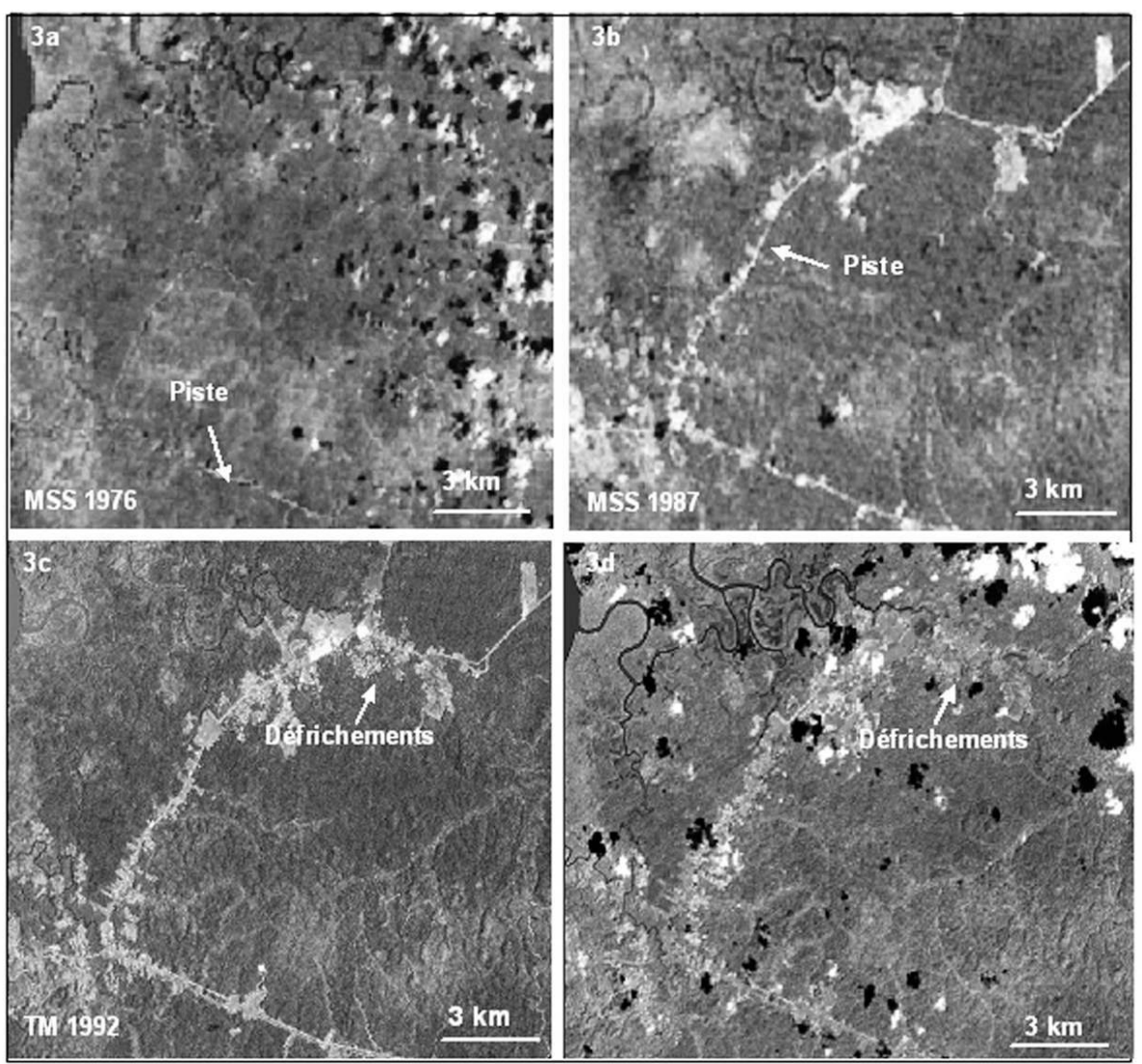

o NASANUS Geological Sunvey pour les extraits MSS et TM; CNES pour lextrait Spot

Photo 3a-3d : Processus et étapes de l'insertion territoriale par les abattis en Guyane française : exemple dans la localité de Charvein au Nord-Ouest

Process and stages of territorial insertion using shifting cultivation in French Guiana: example in the Charvein locality in the North-West

nomique par la régularisation de la situation administrative des immigrés et par la viabilisation et la pérennisation des abattis et autres exploitations agricoles. Dans ce cas, l'abattis joue bien le rôle de vecteur de l'insertion territoriale et permet ensuite à certains immigrés de s’insérer dans les circuits socio-économiques de la région. Cette insertion territoriale par les abattis semble néanmoins embryonnaire et insuffisante pour permettre aux communautés d'immigrés d'établir un rapport de pouvoir ou de force en vue de faire corriger les inégalités sociales en Guyane si on fait une interprétation sur la base de la définition de l'appropriation de l'espace proposée par Ripoll et Veschambre (2005). Avec la croissance démographique, l'insertion territoriale par les abattis se répand de sorte que le nombre d'abattis apparaît de plus en plus comme un révélateur de la pression foncière.

\section{L'IMPORTANCE DES ABATTIS DANS LE PAYSAGE AGRICOLE DE LA GUYANE FRANÇAISE}

À l'image de son territoire caractérisé par le binôme savanes littorales/forêt intérieure, les paysages agraires de la Guyane française se répartissent en deux catégories : d'une part, les paysages d'agriculture dite moderne, résultant essentiellement de la riziculture et de l'élevage bovin, d'autre 


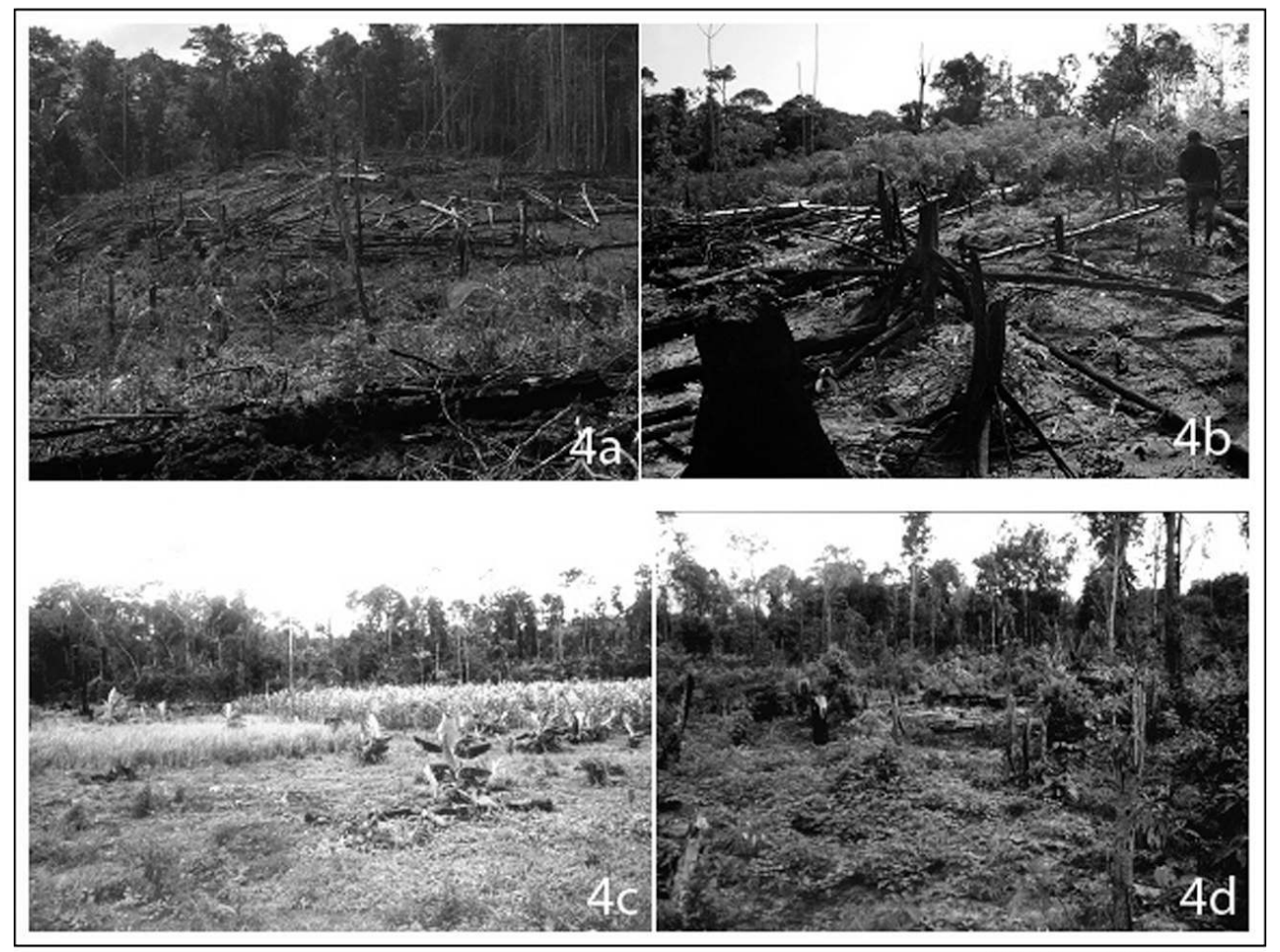

Cliches Tsayem 2000, 2005 et 2006

Photo 4a-4d : Abattis dans la localité de Charvein. En haut, parcelles défrichées et brûlées En bas à gauche, parcelle juxtaposant patates ( $1^{\mathrm{er}}$ plan), maïs (à droite) et friche herbeuse (à gauche). En bas à droite, parcelle de patates douces intercalées entre les troncs d'arbres. Slash and burn cultivation in the Charvein locality

part les paysages d'abattis, résultant de la pratique de l'agriculture traditionnelle itinérante sur brûlis.

L'agriculture moderne a fait l'objet de plusieurs projets de développement dans la zone littorale. Constituée d'exploitations de surface bien plus importante que celle des abattis, cette forme d'agriculture s'est vue assigner deux principaux objectifs : approvisionner la Métropole en produits exotiques et, de plus en plus, satisfaire la demande locale en produits vivriers. Ces projets de développement de l'agriculture moderne ont connu plus ou moins de bonheur. Le plus ambitieux d'entre eux est sans doute le " plan vert » initié en 1975 avec pour objectif de créer 260 exploitations agricoles de 65 ha en moyenne pendant 10 ans, soit au total 1700 ha. C'est surtout l'élevage bovin, la riziculture et, dans une moindre mesure, l'arboriculture fruitière, qui ont bénéficié de ce plan de développement agricole aux résultats très mitigés (Piantoni, 2002).

L'agriculture guyanaise a évolué de manière notable depuis la fin des années 1970. Cette évolution se traduit essentiellement par l'augmentation des surfaces mises en culture. La surface agricole utilisée a triplé au cours de ces 20 dernières années, passant de 7314 ha en 1980 à 23176 ha en 2000 (tableau 4). Durant la même période, la surface des abattis a été multipliée par 5, passant de 1869 ha en 1980 à 8453 ha en 2000 (tableau 4). Les abattis représentent $36 \%$ de la surface agricole utilisée en 2000, contre $25 \%$ en 1980. Cette part paraît faible en raison du fait que les exploitations agricoles de type abattis, contrairement aux exploitations de type riziculture ou pâturage, sont de petites dimensions. Toutefois, lorsqu'on prend comme indicateur le nombre d'exploitations agricoles, l'importance des abattis dans le paysage agricole guyanais s'avère 
considérable. D'après les enquêtes de la direction départementale de l'Agriculture et de la Forêt, les abattis représentent $90 \%$ des exploitations agricoles de la Guyane en 2000, contre $76 \%$ en 1980 (fig. 3). Cette évolution est à mettre en relation avec la multiplication des exploitations dans le nord-ouest, suite à l'immigration enregistrée dans cette région durant ces dernières décennies (Piantoni, 2002).

\begin{tabular}{|l|l|l|l|l|}
\hline \multicolumn{1}{|c|}{ Années } & \multicolumn{1}{|c|}{ SAU (ha) } & $\begin{array}{c}\text { Nombre total d'ex- } \\
\text { ploitations }\end{array}$ & nombre d'abattis & Surface des abattis \\
\hline 1980 & 7314 & 2209 & 1600 & 1869 \\
\hline 1989 & 20645 & 4491 & 3506 & 5593 \\
\hline 2000 & 23176 & 5310 & 4792 & 8453 \\
\hline
\end{tabular}

Figure 3 : Évolution du nombre d'abattis par rapport au nombre total d'exploitations agricoles en Guyane française entre 1980 et 2000 parcels

Evolution of the quantity of shifting cultivation parcels regarding the total number of agricultural

Tableau 4 : Évolution de la surface agricole utilisée (SAU) en Guyane française (Source : Tsayem, 2002)

Evolution of agricultural surfaces in French Guiana

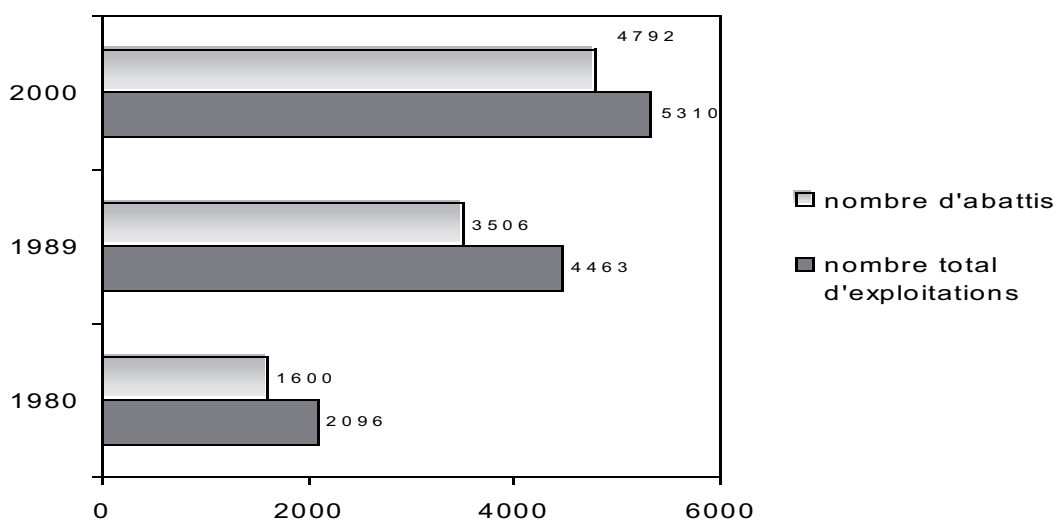

\section{ProblèMes DE GESTION FONCIÈRE ET D’AMÉNAGEMENT DU TERRITOIRE POSÉS PAR LES ABATTIS}

Les abattis posent des problèmes d'aménagement et de gestion foncière car ils relèvent très souvent d'opérations non planifiées. Généralement considérée comme le symptôme de l'occupation anachronique et anarchique de la forêt, la pratique des abattis s'effectue en marge de la légalité et pose le problème de consolidation de la propriété foncière. Les abattis font rarement l'objet de politiques concertées de planification et de gestion de l'espace, alors que le contexte foncier paraît précaire.

L'État est propriétaire de $90 \%$ du patrimoine foncier de la Guyane (tableau 5). Cette situation est paradoxale car les propriétés privées représentent seulement $9 \%$ de l'étendue du territoire contrairement à ce qu'on observe ailleurs, notamment en France métropolitaine où l'État ne détient une infime partie du foncier. De la sorte, la «pénurie des terres » est de plus en plus évoquée en Guyane française. La demande en terres est très forte et continue d'augmenter, en liaison avec l'accroissement démographique et l'immigration. La pression foncière se traduit par la multiplication des occupations sans titre (squatterisation du domaine public), ce qui confine la tenure des terres dans la précarité. Une étude récente de l'Institut d'Émission d'Outre Mer (IEDOM, 2005) indique que $73 \%$ des exploitations agricoles en Guyane française, soit trois exploitations sur quatre, sont exploitées sans titre foncier, ce qui représente $41 \%$ de la surface agricole utilisée (fig. 4). 


\begin{tabular}{|l|l|l|}
\hline \multirow{2}{*}{ Propriétaires } & \multicolumn{2}{|c|}{ Superficies } \\
\cline { 2 - 3 } & (ha) & $\%$ \\
\hline État & 7525800 & 90,4 \\
\hline Particuliers & 784093 & 9,4 \\
\hline Département & 10355 & 0,15 \\
\hline Communes & 4206 & 0,05 \\
\hline Total & 8324274 & 100 \\
\hline
\end{tabular}

Tableau 5 : Répartition du foncier en Guyane française (Source : Taubira- Delannon, 1998) Land distribution in French Guiana

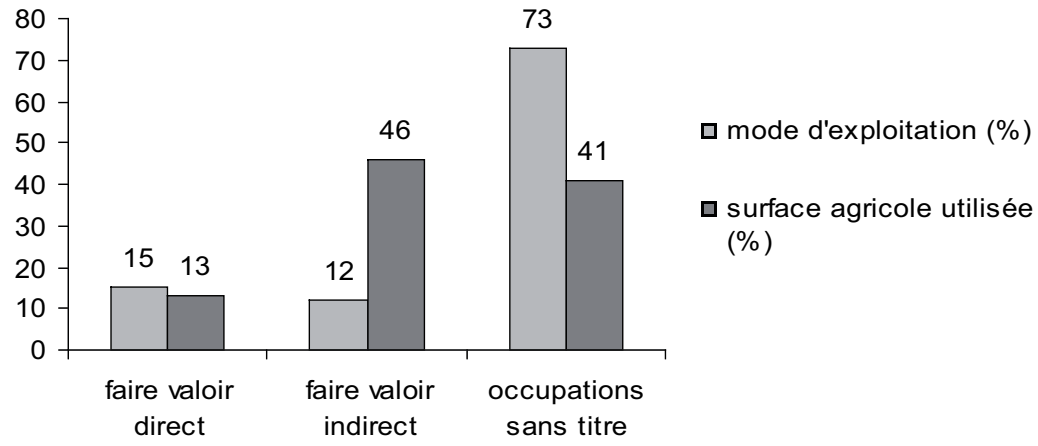

Source :IEDOM 2005
Figure 4 : Importance des occupations sans titre dans les modes d'exploitation agricole en Guyane française Part of non official land use in the agricultural domain in French Guiana

Trois mille demandes d'attributions foncières portant sur 49700 ha ont été enregistrées par le Service des Domaines (Tsayem, 2001). Une surface deux fois supérieure à celle qui est déjà en exploitation est donc convoitée, ce qui signifie que la surface agricole totale pourrait passer du simple au triple si toutes les demandes sont acceptées.

La législation foncière (code du domaine de l'État) prévoit deux principaux modes d'attribution gratuite des parcelles du domaine privé de l'État pour un usage agricole en Guyane française : la concession et le bail emphytéotique. La concession concerne les parcelles de 5 ha au maximum. Elle est accordée pour une durée de 5 ans aux particuliers qui en font la demande. Si la «mise en valeur agricole » effective est constatée au bout de cette période de 5 ans, la parcelle peut être cédée à l'utilisateur qui acquiert de ce fait un droit de propriété. Quant au bail emphytéotique, il est accordé pour les exploitations agricoles de surface unitaire supérieure à 5 ha. Il confère le droit d'usage de la parcelle pour une période de 30 ans, au terme de laquelle le terrain redevient la propriété de l'État.

Pour les communautés qui tirent traditionnellement leurs moyens de subsistance de la forêt, les zones de droit d'usage collectif leur sont attribuées par les pouvoirs publics en concertation avec ces communautés. Il s'agit en particulier des groupes d'Amérindiens et de Noirs Marrons. Le droit qui leur est ainsi accordé collectivement ne concerne que l'usage des terres et non l'appropriation. Nos observations de terrain montrent que cette nuance n'est pas bien comprise par tous, car dans certaines communautés, notamment chez les Noirs Marrons, on considère que lorsqu'une famille défriche une parcelle jamais cultivée auparavant, elle en détient une sorte de droit de propriété. En vertu de ce «droit », cette famille peut rétrocéder la parcelle à sa descendance, et aucune autre famille ne peut prétendre à ladite parcelle sans en référer à la première. C'est une sorte de droit coutumier qu'on retrouve dans la plupart des sociétés africaines. Ainsi se juxtaposent le droit 
coutumier et le droit régalien, ce qui rend plus complexes les problèmes de gestion foncière au regard de l'augmentation actuelle des besoins en terres agricoles.

Par ailleurs, les autorités administratives accordent souvent un droit tacite à certaines populations, notamment aux immigrés, pour qu'elles défrichent et cultivent les abattis non loin des routes pour satisfaire leurs besoins de subsistance. Dans une telle situation, l'abattis est une forme d'insertion territoriale permettant à une certaine catégorie de population, d'afficher sa présence et de bénéficier éventuellement de la reconnaissance de l'État comme le montre l'exemple de Charvein.

Les problèmes de pression foncière et d'aménagement de l'espace en Guyane française ont conduit l'État à créer en 1996, un organisme chargé de viabiliser, de restructurer le domaine privé de l'État et de régulariser la situation foncière. Il s'agit de l'EPAG (Établissement Public d’Aménagement en Guyane). Ses opérations d'aménagement foncier ont débuté en avril 1998 et consistaient notamment en l'instruction des demandes d'attribution foncière enregistrées par le Service des Domaines. La viabilisation et l'attribution des parcelles pour les abattis faisaient donc parti des missions de l'EPAG. Mais après quelques années de fonctionnement, les résultats escomptés n'ont pas été obtenus, de sorte que le problème d'attribution et de gestion du foncier agricole continuait de se poser avec acuité. Ces résultats très mitigés s'expliquent par plusieurs facteurs : la complexité et les difficultés d'application de la législation foncière en Guyane française; l'absence de clarté et de consensus sur les politiques locales d'accès au foncier; la forte pression sociale sous-tendue par des revendications portées par des associations; les divergences d'analyses et de prises de décisions entre les autorités publiques, les élus locaux, les responsables de l'EPAG et des autres services de l'État (DDAF, ONF, etc.). Les opérations de régularisation du foncier agricole sont effectuées depuis quelques années par le CNASEA (Centre National pour l'Aménagement des Structures des Exploitations Agricoles). Un volet important de ces opérations concerne les occupations illégales du domaine privé de l'État. L'ordonnance du 2 septembre 1998, complétée par deux décrets d'application du 11 mars 2000, définissent les modalités de cette régularisation. Il est prévu de céder gratuitement $2500 \mathrm{~m}^{2}$ à chaque agriculteur qui ne détient aucun droit de propriété et/ou d'usage de la parcelle qu'il a défrichée avant le 4 septembre 1998. L'application de cette disposition n'est pas évidente, en raison de la difficulté de faire un inventaire précis de l'occupation du sol à cette date. Nos travaux précédents fondés sur l'utilisation d'images satellites et de photographies aériennes nous amènent à suggérer l'utilisation des données de télédétection, complétées par les enquêtes et les relevés de terrain, pour mieux traiter les problèmes d'aménagement liés aux défrichements agricoles en Guyane (Tsayem, 2002; Tsayem et al., 2002 et 2001 ; Tsayem, 1999).

\section{Conclusion}

La gestion du foncier et l'aménagement du territoire ne sont pas aisés dans un contexte d'occupation agricole précaire. La tâche est d'autant plus complexe que les abattis sont spontanés et itinérants, et ne font généralement pas l'objet de planification. Les exemples présentés dans cet article montrent que dans le contexte guyanais caractérisé par une forte croissance démographique alimentée en partie par l'immigration, la demande en terres agricoles augmente rapidement au point de susciter une pression foncière qui peut paraître paradoxale compte tenu des faibles densités démographiques générales. Mais la spécificité majeure du foncier en Guyane (environ $90 \%$ du foncier constitué de forêt appartenant au domaine privé de l'État et géré par l'Office National des Forêts) atténue le caractère paradoxal de cette pression foncière qui s'exerce essentiellement aux alentours des points d'ancrage que sont les villes, les bourgs, les pistes et les cours d'eau.

Le nombre important des abattis leur confère un rôle qui va bien au-delà de la fonction agricole. Ces abattis remplissent aussi des fonctions sociales, culturelles, économiques et environnementales (Tsayem et Manusset, 2008 ; Manusset, 2004) tout en jouant un rôle important dans l'ancrage 
territorial des populations autochtones et immigrées. Les abattis deviennent de plus en plus incontournables dans les politiques publiques d'aménagement du territoire et de gestion foncière même si l'intervention des organismes d'aménagement du territoire et de gestion foncière se fait souvent après coup pour tenter de restructurer l'espace. Il s'agit alors de réaménagement et non plus seulement d'aménagement.

En tant que moyen d'insertion territoriale des populations généralement sans grands moyens financiers et matériels (communautés d'Amérindiens et de Noirs Marrons, immigrés), les abattis devraient être davantage pris en compte non seulement dans les politiques de gestion du territoire, mais aussi dans les politiques sociales et dans les dispositifs d'aides aux populations locales. En effet, il faudrait pouvoir considérer l'abattis non seulement comme une pratique agricole, mais aussi comme un vecteur de territorialité dans la mesure où l'abattis permet de se fixer à un endroit et d'y cultiver des produits agricoles pour la subsistance alimentaire et pour la commercialisation qui est source d'un complément de revenus. L'abattis apparaît ainsi à la base d'un processus d'ancrage territorial et de participation à la vie socio-économique locale.

\section{Bibliographie}

Fournier (J.-M.), 2007. - « Géographie sociale et territoires, de la confusion sémantique à l'utilité sociale? », Travaux et Documents ESO, n 26, p. 29-35, dossier « le territoire en géographie sociale ».

Gachet (J.-P.), Toulemonde (M.), Garganta (E.), 1993. - « Guyane française : une mini-frontière amazonienne. Mouvements migratoires et transformation des systèmes de production agricole », dans LiNCK (T.) (dir.), Agricultures et paysanneries en Amérique Latine. Mutations et Recompositions, Paris, ORSTOM éditions, p. 187-189.

Grenand (P.), Grenand (F.), 1997. - L'occupation amérindienne : ethnoarchéologie, ethnohistoire, dans Mazière (M.) (dir.), L'archéologie en Guyane, Cayenne, édition APPAAG, p. 58-71.

HÉRIN (R.), 2007. - «À propos du territoire et des réticences d'usage qu’il suscite », Travaux et Documents $E S O, n^{\circ} 26$, p. 17-28, dossier « le territoire en géographie sociale ».

IEDOM, 2004. - La Guyane en 2004. Cayenne, édition IEDOM, 162 p.

-, 2005. - Le foncier agricole en Guyane. Cayenne, édition IEDOM, Note expresse, 4 p., [http://www.iedom. fr/doc/NotExp26_Guyane_foncierAgricole.pdf].

INSEE-ACSE, 2006. - Atlas des populations immigrées en Guyane, Cayenne, INSEE Antilles-Guyane, 28 p. [http://www.insee.fr/fr/insee_regions/guyane/publi/Atlas_immigres_gy.pdf].

Keerle (R.), Sechet (R.), 2007. - «Petite histoire de nos délicatesses avec le territoire », Travaux et Documents ESO, n²6, p. 7-15, dossier « le territoire en géographie sociale».

Le Tourneau (F.-M.), 2002. - « La représentation du peuplement en pays pionnier : l'Amazonie brésilienne », L'Espace Géographique, 2002-2, p. 145-152.

Manusset (S.) 1999. - « La question des abattis à Saint-Georges de l'Oyapock en Guyane française », dans Bahuchet (S.), Bley (D.), Pagezy (H.), Vernazza-Licht (dir.), L'homme et la forêt tropicale, Marseille, société d'écologie humaine, p. 267-279.

Manusset (S.) 2004. - La question des abattis dans le bas Oyapock (Guyane) : pérennité et diversité d'une pratique agricole en forêt tropicale et en contexte multiculturel. Thèse de Doctorat, Aix-en-Provence, Université Aix-Marseille 3, 355 p.

Moine (A.), 2006. - « Le territoire comme un système complexe : un concept opératoire pour l'aménagement et la géographie », L’Espace Géographique, 2006-2, p. 115-132.

PiAnTONi (F.) 1998. - «Rupture territoriale et comportements démographiques endogènes : processus de territorialisation de l'espace frontalier Maroni (Surinam/Guyane française) depuis 1986 », communication présentée au colloque international de l'Association des Démographes de Langue Française, La Rochelle, 22-26 septembre 1998, 15 p. 
—, 1999. - « La migration surinamaise dans le nord-ouest guyanais : analyse du processus de territorialisation du milieu rural depuis 1986 », communication présentée au symposium international Migration : Nation, Place and Territorial Dynamics, 19-25 avril 1999, Sao Paulo, Brasil, 15 p.

—, 2002. Pouvoir national et acteurs locaux : l'enjeu des mobilités dans un espace en marges. Le cas de la Guyane française. Thèse de Doctorat, Poitiers, Université de Poitiers, 474 p.

Poissonnet (M.), Parmantier (A.), Wyngaarde (B.), Bifane (E.-E.), Deménois (J.), Lescuyer (G.), 2006. - « Espoirs et difficultés du transfert de la gestion forestière à deux communautés de Guyane et du Cameroun », Bois et Forêts des Tropiques, n² 289 (3), p. 5-16.

Ripoll (F.), Veschambre (V.) (textes réunis par), 2005. - « L'appropriation de l'espace : sur la dimension des inégalités sociales et des rapports de pouvoir », Norois, $n^{\circ}$ 195-2.

Taubira-Delannon (C.), 1998. - La question foncière en Guyane : retranscription de la visioconférence du 18 septembre 1998, Cayenne, 70 p.

TsaYem (M.), 1999. - « SIG et cartographie de l'occupation du sol : l'exemple de Saint-Georges de l'Oyapock en Guyane française ", Science et Changements Planétaires/Sécheresse, vol. 4, nº 10, p. 289-295.

Tsayem Demaze (M.), 2002. - Caractérisation et suivi de la déforestation en milieu tropical par télédétection: application aux défrichements agricoles en Guyane française et au Brésil, Thèse de Doctorat, Université d'Orléans, 242 p.

Tsayem Demaze (M.), Polidori (L.), Fotsing (J.-M.), 2001. - « Caractérisation multi-échelle et multi-capteur de la déforestation tropicale amazonienne », Bulletin de la Société Française de Photogrammétrie et Télédétection, $\mathrm{n}^{\circ} 161$, p. 74-84.

Tsayem Demaze (M.), Fotsing (J.-M.), Huynh (F.), 2002 - « «a déforestation dans la région de Saint-Georges de l'Oyapock (Guyane française) », Les Cahiers d'Outre Mer, 55 (218), mars-juin 2002, p. 197-222.

Tsayem Demaze (M.), Manusset (S.), 2008. - « Modifications en cours dans l'agriculture itinérante sur brûlis en Guyane française : vers la fin de la durabilité écologique et socio-culturelle? » (à paraître dans Les Cahiers d'Outre Mer, $\mathrm{n}^{\circ}$ 241-242).

Cet article a été reçu le le 5 novembre 2007 et définitivement accepté le 10 avril 2008. 
